PROCEEDINGS OF THE

AMERICAN MATHEMATICAL SOCIETY

Volume 139, Number 10, October 2011, Pages 3719-3726

S 0002-9939(2011)10822-0

Article electronically published on February 24, 2011

\title{
ON THE EQUALITY CONDITIONS OF THE BRUNN-MINKOWSKI THEOREM
}

\author{
DANIEL A. KLAIN
}

(Communicated by Thomas Schlumprecht)

\begin{abstract}
This article describes a new proof of the equality condition for the Brunn-Minkowski inequality. The Brunn-Minkowski Theorem asserts that, for compact convex sets $K, L \subseteq \mathbb{R}^{n}$, the $n$-th root of the Euclidean volume $V_{n}$ is concave with respect to Minkowski combinations; that is, for $\lambda \in[0,1]$,

$$
V_{n}((1-\lambda) K+\lambda L)^{1 / n} \geq(1-\lambda) V_{n}(K)^{1 / n}+\lambda V_{n}(L)^{1 / n} .
$$
\end{abstract}

The equality condition asserts that if $K$ and $L$ both have positive volume, then equality holds for some $\lambda \in(0,1)$ if and only if $K$ and $L$ are homothetic.

Denote $n$-dimensional Euclidean space by $\mathbb{R}^{n}$. Given compact convex subsets $K, L \subseteq \mathbb{R}^{n}$ and $a, b \geq 0$, denote

$$
a K+b L=\{a x+b y \mid x \in K \text { and } y \in L\} .
$$

An expression of this form is called a Minkowski combination or Minkowski sum. Since $K$ and $L$ are convex sets, the set $a K+b L$ is also convex. Convexity also implies that $a K+b K=(a+b) K$ for all $a, b \geq 0$, although this does not hold for general sets. Two sets $K$ and $L$ are homothetic if $K=a L+x$ for some $a>0$ and some point $x \in \mathbb{R}^{n}$. The $n$-dimensional (Euclidean) volume of $K$ will be denoted by $V_{n}(K)$.

The Brunn-Minkowski Theorem asserts that the $n$-th root of the Euclidean volume $V_{n}$ is concave with respect to Minkowski combinations; that is, for $\lambda \in[0,1]$,

$$
V_{n}((1-\lambda) K+\lambda L)^{1 / n} \geq(1-\lambda) V_{n}(K)^{1 / n}+\lambda V_{n}(L)^{1 / n} .
$$

If $K$ and $L$ have non-empty interiors, then equality holds for some $\lambda \in(0,1)$ if and only if $K$ and $L$ are homothetic. This article describes a new proof of this equality condition, using a homothetic projection theorem of Hadwiger.

The Brunn-Minkowski Theorem is the centerpiece of modern convex geometry 1, 6. 14, 15. This theorem encodes as special cases the classical isoperimetric inequality (relating volume and surface area [14, p. 318]), Urysohn's inequality (relating volume and mean width, and strengthening the isodiametric inequality [14, p. 318]), and families of inequalities relating mean projections [14, p. 333]. The concavity implied by (11) leads to families of second-order discriminant-type inequalities for mixed volumes, such as Minkowski's second mixed volume inequality [14, p. 317] and (after substantial additional labor) the Alexandrov-Fenchel

Received by the editors May 9, 2010 and, in revised form, September 2, 2010.

2010 Mathematics Subject Classification. Primary 52A20, 52A38, 52A39, 52A40.

(C)2011 American Mathematical Society Reverts to public domain 28 years from publication 
inequality [14, p. 327], a difficult and far-reaching result with consequences in geometric analysis, combinatorics, and algebraic geometry [4]. Analytic generalizations of the Brunn-Minkowski theorem include the Prékopa-Leindler inequality [3, 10]. The Brunn-Minkowski theorem also serves as the starting point for analogous developments such as the dual Brunn-Minkowski theory for star-shaped sets [11, the $L_{p}$-Brunn-Minkowski theory [12, capacitary Brunn-Minkowski inequalities [2], and Brunn-Minkowski inequalities for integer lattices [7. Of special interest are the equality conditions for (1), which imply, for example, the uniqueness of solutions to the Minkowski problem relating convex bodies to measures on the unit sphere 1, 14. A recent and comprehensive survey on the Brunn-Minkowski inequality and its variations, applications, extensions, and generalizations can be found in [5].

There are many ways to prove the Brunn-Minkowski Theorem. For the case of compact convex sets, Kneser and Süss used an induction argument on dimension via slicing [14, p. 310]. This proof first verifies the inequality (11) and then addresses the equality case with a more subtle argument.

Hadwiger and Ohmann give a more general proof by using the concavity of the geometric mean to prove (11) for the case of rectangular boxes and follow with a divide-and-conquer argument that extends (1) to finite unions of boxes. They conclude with an approximation step that verifies (11) for all measurable sets 9] (see also [5 and [16, p. 297]). Hadwiger and Ohmann also show that if equality holds in (1), then $K$ and $L$ must both be compact convex sets with at most a set of measure zero removed. Therefore, the question of equality in (1) is addressed completely (up to measure zero) by the case of compact convex sets.

Another especially intuitive proof of the inequality (1) for compact convex sets uses Steiner symmetrization [5, 16]; however, this method relies on approximation and gives no insight into the equality conditions.

In contrast to earlier methods, the proof of the equality condition for (11) presented in this note uses orthogonal projection rather than slicing. Sections 1, 2, and 3 provide some technical background. The new proof is presented in Section 4 .

\section{BACKGROUND}

Let $\mathscr{K}_{n}$ denote the set of compact convex subsets of $\mathbb{R}^{n}$. If $u$ is a unit vector in $\mathbb{R}^{n}$, denote by $K_{u}$ the orthogonal projection of a set $K$ onto the subspace $u^{\perp}$. More generally, if $\xi$ is a $d$-dimensional subspace of $\mathbb{R}^{n}$, denote by $K_{\xi}$ the orthogonal projection of a set $K$ onto the subspace $\xi$. The boundary of a compact convex set $K$ relative to its affine hull will be denoted by $\partial K$.

Let $h_{K}: \mathbb{R}^{n} \rightarrow \mathbb{R}$ denote the support function of a compact convex set $K$; that is,

$$
h_{K}(v)=\max _{x \in K} x \cdot v .
$$

The standard separation theorems of convex geometry imply that the support function $h_{K}$ characterizes the body $K$; that is, $h_{K}=h_{L}$ if and only if $K=L$. If $\xi$ is a subspace of $\mathbb{R}^{n}$, then the support function of $K_{\xi}$ within the subspace $\xi$ is given by the restriction of $h_{K}$ to $\xi$. Support functions satisfy the identity $h_{a K+b L}=a h_{K}+b h_{L}$. (See, for example, any of [1, 14, 16.)

If $u$ is a unit vector in $\mathbb{R}^{n}$, denote by $K^{u}$ the support set of $K$ in the direction of $u$; that is,

$$
K^{u}=\left\{x \in K \mid x \cdot u=h_{K}(u)\right\} .
$$


If $P$ is a convex polytope, then $P^{u}$ is the maximal face of $P$ having $u$ in its outer normal cone.

Given $K, L \in \mathscr{K}_{n}$ and $\varepsilon>0$, the function $V_{n}(K+\varepsilon L)$ is a polynomial in $\varepsilon$, whose coefficients are given by Steiner's formula [1, 14, 16]. In particular, the following derivative is well defined:

$$
n V_{n-1,1}(K, L)=\lim _{\varepsilon \rightarrow 0} \frac{V_{n}(K+\varepsilon L)-V_{n}(K)}{\varepsilon}=\left.\frac{d}{d \varepsilon}\right|_{\varepsilon=0} V_{n}(K+\varepsilon L) .
$$

The expression $V_{n-1,1}(K, L)$ is an example of a mixed volume of $K$ and $L$. Since the volume of any set is invariant under translation, it follows from the definition (2) that, for any point $p \in \mathbb{R}^{n}$,

$$
V_{n-1,1}(K+p, L)=V_{n-1,1}(K, L+p)=V_{n-1,1}(K, L) .
$$

If $P$ is a polytope, then the mixed volume $V_{n-1,1}(P, K)$ satisfies the classical "base-height" formula

$$
V_{n-1,1}(P, K)=\frac{1}{n} \sum_{u \perp \partial P} h_{K}(u) V_{n-1}\left(P^{u}\right),
$$

where this sum is finite, taken over all outer unit normals $u$ to the facets on the boundary $\partial P$. Since compact convex sets can be approximated (in the Hausdorff metric) by convex polytopes, the equation (3) implies that, for $K, L, M \in \mathscr{K}_{n}$ and $a, b \geq 0$,

- $V_{n-1,1}(K, L) \geq 0$,

- $V_{n-1,1}(K, K)=V_{n}(K)$,

- $V_{n-1,1}(a K, b L)=a^{n-1} b V_{n-1,1}(K, L)$,

- $V_{n-1,1}(K, L+M)=V_{n-1,1}(K, L)+V_{n-1,1}(K, M)$,

where the final identity follows from (3) and the linearity of support functions with respect to Minkowski sums. For $n \geq 3$, the function $V_{n-1,1}$ is not typically symmetric in its parameters: usually $V_{n-1,1}(K, L) \neq V_{n-1,1}(L, K)$. In particular, the fourth (linearity) property above does not typically hold for the first parameter of $V_{n-1,1}$.

Important special (and more well-known) cases of mixed volumes result from suitable choices of $K$ or $L$. For example, if $B$ is the unit ball, centered at the origin, then (3) implies that $n V_{n-1,1}(P, B)$ gives the surface area of $P$. A limiting argument then yields the same fact for $n V_{n-1,1}(K, B)$, where $K$ is any compact convex set. Similar arguments imply that, if $\overline{o u}$ denotes the line segment with endpoints at $o$ and a unit vector $u$, then

$$
n V_{n-1,1}(K, \overline{o u})=V_{n-1}\left(K_{u}\right),
$$

the $(n-1)$-volume of the corresponding orthogonal projection of $K$. These and many other properties of convex bodies and mixed volumes are described in each of 1, 14, 16.

One especially intuitive proof of the Brunn-Minkowski inequality (1) for compact convex sets uses Steiner symmetrization. Given a unit vector $u$, view $K$ as a family of line segments parallel to $u$. Slide these segments along $u$ so that each is symmetrically balanced around the hyperplane $u^{\perp}$. By Cavalieri's principle, the volume of $K$ is unchanged. Call the new set $s t_{u}(K)$. It is not difficult to show that $s t_{u}(K)$ is also convex, and that $s t_{u}(K)+s t_{u}(L) \subseteq s t_{u}(K+L)$. A little more work verifies the following intuitive assertion: if you iterate Steiner symmetrization 
of $K$ through a suitable sequence of directions, these iterations tend to round out the body $K$ to a Euclidean ball $B_{K}$ having the same volume as the original set $K$. Meanwhile, it follows from the aforementioned superadditivity relation that $B_{K}+B_{L} \subseteq B_{K+L}$, so that

$$
\begin{aligned}
V_{n}(K+L)^{1 / n}=V_{n}\left(B_{K+L}\right)^{1 / n} & \geq V_{n}\left(B_{K}+B_{L}\right)^{1 / n} \\
& =V_{n}\left(B_{K}\right)^{1 / n}+V_{n}\left(B_{L}\right)^{1 / n}=V_{n}(K)^{1 / n}+V_{n}(L)^{1 / n} .
\end{aligned}
$$

The technical details behind Steiner symmetrization and the proof outlined above can be found in [16, pp. 306-314]. Once again, because of an approximation step (taking the limit of a sequence of Steiner symmetrizations), it is not clear from this proof exactly when equality would hold in (11).

This matter is addressed by the following theorem.

Theorem 1.1 (Minkowski). If $K$ and $L$ are compact convex sets with non-empty interiors, then equality holds in (11) if and only if $K$ and $L$ are homothetic.

While homothety is evidently sufficient, its necessity is far from obvious.

Simple arguments show that the Brunn-Minkowski inequality (11) is equivalent to Minkowski's mixed volume inequality,

$$
V_{n-1,1}(K, L)^{n} \geq V_{n}(K)^{n-1} V_{n}(L),
$$

where the equality conditions are the same as for (11).

Note that (5) is trivial if either $V_{n}(K)=0$ or $V_{n}(L)=0$. Moreover, both sides of (5D) are positively homogeneous of degree $n(n-1)$ with respect to scaling the body $K$ and positively homogeneous of degree $n$ with respect to scaling the body $L$. It follows that, for $K$ and $L$ with non-empty interiors, the inequality (5) is equivalent to the assertion that

$$
V_{n-1,1}(K, L) \geq 1
$$

whenever $V_{n}(K)=V_{n}(L)=1$.

To prove ([6), and the equivalent (5), using (10), suppose that $V_{n}(K)=V_{n}(L)=1$, and let

$$
f(t)=V_{n}((1-t) K+t L)=(1-t)^{n} V_{n}\left(K+\frac{t}{1-t} L\right)
$$

for $t \in[0,1)$. By (2) and the chain rule,

$$
f^{\prime}(0)=-n V_{n}(K)+n V_{n-1,1}(K, L)=-n+n V_{n-1,1}(K, L)
$$

Since $f^{1 / n}$ is concave by (1), we have $f \geq 1$ on $[0,1)$, while $f(0)=1$, so that $f^{\prime}(0) \geq 0$, and $V_{n-1,1}(K, L) \geq 1$.

If equality holds in (11) for any value of $t \in(0,1)$, the concavity of $f^{1 / n}$ implies that $f^{1 / n}$ must be linear. Since $f(0)=f(1)=1$ by construction, the function $f^{1 / n}$ is constant. It follows that $f^{\prime}(0)=0$, so that $V_{n-1,1}(K, L)=1$ and equality holds in (6).

To prove (11) using (5) the argument is even simpler. Denote $K_{t}=(1-t) K+t L$. Then

$$
\begin{aligned}
V_{n}\left(K_{t}\right)=V_{n-1,1}\left(K_{t}, K_{t}\right) & =(1-t) V_{n-1,1}\left(K_{t}, K\right)+t V_{n-1,1}\left(K_{t}, L\right) \\
& \geq(1-t) V_{n}\left(K_{t}\right)^{\frac{n-1}{n}} V(K)^{\frac{1}{n}}+t V_{n}\left(K_{t}\right)^{\frac{n-1}{n}} V(L)^{\frac{1}{n}},
\end{aligned}
$$

by two applications of (5). The inequality (1) then follows after division by $V_{n}\left(K_{t}\right)^{\frac{n-1}{n}}$. 
Suppose equality holds in (5), or equivalently, in (6). The non-negative function $f^{1 / n}$ defined by (7) is concave on the interval $[0,1]$ by (1). Since $f(0)=f(1)=1$, the function $f^{1 / n} \geq 1$ on $[0,1]$ by concavity. But equality in (6), together with the identity (8), implies that $f^{1 / n}$ has zero derivative at $t=0$. By concavity again, it follows that $f^{1 / n}$ has a non-positive derivative and must be non-increasing on $[0,1]$. In other words, $f^{1 / n}$ is constant and equality holds in (1).

For a more complete discussion, see any of [1, 5, 14, 16].

\section{MiXed AREA}

Denote the special case of 2-dimensional volume $V_{2}$ by $A$ for area, and denote $V_{1,1}(K, L)$ by $A(K, L)$, the mixed area. Unlike the higher dimensional mixed volumes, the mixed area is symmetric in its parameters: $A(K, L)=A(L, K)$. If $\Delta$ is a triangle with outward edge unit normals $u_{1}, u_{2}, u_{3}$, then

$$
A(K, \Delta)=A(\Delta, K)=\frac{1}{2} \sum_{i} h_{K}\left(u_{i}\right)\left|\Delta^{u_{i}}\right|
$$

where $\left|\Delta^{u_{i}}\right|$ denotes the length of the $i$-th edge of the triangle. This identity leads to the following proposition.

Proposition 2.1. Let $K, L \in \mathscr{K}_{2}$, and suppose that

$$
A(K, \Delta)=A(L, \Delta)
$$

for every triangle $\Delta$ in $\mathbb{R}^{2}$. Then $K$ and $L$ are translates.

Proof. Translate $K$ and $L$ so that both lie in the first quadrant of $\mathbb{R}^{2}$ and are supported by the coordinate axes. If $e_{1}$ and $e_{2}$ respectively denote the unit vectors along the two coordinate axes, we now have $h_{K}\left(-e_{1}\right)=h_{K}\left(-e_{2}\right)=0$, and similarly for $L$. Since mixed area is invariant under translation, the identity (10) still holds for every triangle $\Delta$.

If $u$ is a unit vector with positive coordinates, let $\Delta$ be a right triangle having outward unit normals $-e_{1},-e_{2}, u$. Since $h_{K}\left(-e_{i}\right)=h_{L}\left(-e_{i}\right)=0$, it follows from (9) and (10) that $h_{K}(u)=h_{L}(u)$.

If $u$ is a unit vector in one of the other three quadrants, a similar argument is then made (using a triangle with unit normals $u$, one of the $-e_{i}$ 's, and a suitable choice from the first quadrant) to show that $h_{K}(u)=h_{L}(u)$ once again. It follows that $h_{K}=h_{L}$, so that $K=L$ after the initial translations of $K$ and $L$ into the positive quadrant.

\section{Bodies With homothetiC PRojections ARE Homothetic}

In Section 4 we give a proof of the equality case for (1) and (5) using a projection argument that relies on the following elementary theorem of Hadwiger 8 .

Theorem 3.1 (The Homothetic Projection Theorem). Suppose that $K, L \in \mathscr{K}_{n}$ have non-empty interiors, where $n \geq 3$. If $K_{u}$ and $L_{u}$ are homothetic for all unit vectors $u$, then $K$ and $L$ are homothetic as well.

For completeness of presentation, here is an elementary proof of Theorem 3.1 due to Rogers [13]. 
Proof. Let $e_{i}=(0, \ldots, 1, \ldots, 0)$ denote the unit vector having unit $i$ th coordinate, for $i=1, \ldots, n$.

Translate and scale $K$ and $L$ so that both sets are supported by the positive coordinate halfspace $\left(e_{n}^{\perp}\right)^{+}$, and moreover so that $K_{e_{n}}=L_{e_{n}}$. The latter is possible, because we are given that $K_{e_{n}}$ and $L_{e_{n}}$ are initially homothetic.

It remains to show that, after these translations and dilations, we have $K=L$.

To show this, let $u \in e_{n}^{\perp}$ be a unit vector. Recall from the hypothesis of the theorem that $K_{u}=a L_{u}+v$ for some $a>0$ and some $v \in u^{\perp}$, where both $a$ and $v$ are dependent on $u$.

Let $\xi=\operatorname{Span}\left\{u, e_{n}\right\}^{\perp}=u^{\perp} \cap e_{n}^{\perp}$. Since $\xi \subseteq e_{n}^{\perp}$ and $K_{e_{n}}=L_{e_{n}}$, it follows that $L_{\xi}=K_{\xi}$. Since $\xi \subseteq u^{\perp}$ as well, we also have $K_{\xi}=a L_{\xi}+v_{\xi}$, so that $L_{\xi}=a L_{\xi}+v_{\xi}$.

Since $n \geq 3$, we have $\operatorname{dim} \xi=n-2 \geq 1$. Let $V_{n-2}$ denote volume in $\mathbb{R}^{n-2}$. Since translation does not change volume,

$$
V_{n-2}\left(L_{\xi}\right)=V_{n-2}\left(a L_{\xi}+v_{\xi}\right)=a^{n-2} V_{n-2}\left(L_{\xi}\right) .
$$

Since $L$ has non-empty interior, $V_{n-2}\left(L_{\xi}\right)>0$. It follows that $a=1$ and $v_{\xi}=o$. This implies that $v \in \xi^{\perp}=\operatorname{Span}\left\{u, e_{n}\right\}$; that is, $v=b u+c e_{n}$ for some $b, c \in \mathbb{R}$. Moreover $v \cdot u=0$, since we assumed $v \in u^{\perp}$ to begin with. It follows that $v=c e_{n}$ for some $c \in \mathbb{R}$.

The positive coordinate halfspace $\left(e_{n}^{\perp}\right)^{+}$supports both $K$ and $L$, so that $h_{K}\left(-e_{n}\right)$ $=h_{L}\left(-e_{n}\right)=0$. Since $K_{u}=L_{u}+v$ and $-e_{n} \in u^{\perp}$, we have

$$
0=h_{K}\left(-e_{n}\right)=h_{K_{u}}\left(-e_{n}\right)=h_{L_{u}}\left(-e_{n}\right)+v \cdot\left(-e_{n}\right)=h_{L}\left(-e_{n}\right)-c=-c,
$$

so that $v=c e_{n}=0$, and $K_{u}=L_{u}$.

We have shown that $K_{u}=L_{u}$ for all $u \in e_{n}^{\perp}$. If $w$ is a unit vector, then $w^{\perp} \cap e_{n}^{\perp} \neq \emptyset$, so $w \in u^{\perp}$ for some $u \in e_{n}^{\perp}$. It now follows that

$$
h_{K}(w)=h_{K_{u}}(w)=h_{L_{u}}(w)=h_{L}(w) .
$$

In other words, $h_{K}(w)=h_{L}(w)$ for every unit vector $w$, so that $K=L$.

\section{COnditions For EQUality}

We now have the tools to verify the equality condition for (11) and (5).

Proof of the Equality Condition. For $\lambda \in[0,1]$, denote $K_{\lambda}=(1-\lambda) K+\lambda L$. Suppose that equality holds in (11) for some $\lambda_{0} \in(0,1)$, where $K$ and $L$ have non-empty interiors. Since equality holds for (1) if and only if equality holds for (5) and (6), the homogeneity of mixed volumes allows us to assume without loss of generality that

$$
V_{n}(K)=V_{n}(L)=V_{n}\left(K_{\lambda_{0}}\right)=V_{n-1,1}\left(K_{\lambda_{0}}, L\right)=1 .
$$

The concavity of $V_{n}^{1 / n}$ then implies that

$$
V_{n}\left(K_{\lambda}\right)=1 \text { for all } \lambda \in[0,1] .
$$

Fix a value of $\lambda \in[0,1]$, and suppose $M \in \mathscr{K}_{n}$ is such that $V_{n-1,1}\left(K_{\lambda}, M\right) \leq 1$. Since $V_{n-1,1}(\cdot, \cdot)$ is Minkowski linear in its second parameter, we have

$$
V_{n-1,1}\left(K_{\lambda},(1-x) L+x M\right)=(1-x) V_{n-1,1}\left(K_{\lambda}, L\right)+x V_{n-1,1}\left(K_{\lambda}, M\right) \leq 1
$$


for all $x \in[0,1]$. Since $V_{n}\left(K_{\lambda}\right)=1$, it follows from the inequality (5) that

$$
\begin{aligned}
f(x) & =V_{n}((1-x) L+x M) \\
& =V_{n}((1-x) L+x M) V_{n}\left(K_{\lambda}\right)^{n-1} \\
& \leq V_{n-1,1}\left(K_{\lambda},(1-x) L+x M\right)^{n} \leq 1,
\end{aligned}
$$

for all $x \in[0,1]$. Since $f(0)=V_{n}(L)=1$, it follows that $f^{\prime}(0) \leq 0$. On computing $f^{\prime}(0)$ as in (8), we have $V_{n-1,1}(L, M) \leq 1$.

We have shown that if $V_{n-1,1}\left(K_{\lambda}, M\right) \leq 1$, then $V_{n-1,1}(L, M) \leq 1$. If $M$ is a singleton, then $V_{n-1,1}\left(K_{\lambda}, M\right)=V_{n-1,1}(L, M)=0$. If $M$ is not a singleton, then $V_{n-1,1}\left(K_{\lambda}, M\right)>0$, since $K_{\lambda}$ has an interior. Let $\mu=V_{n-1,1}\left(K_{\lambda}, M\right)$. Then

$$
V_{n-1,1}\left(K_{\lambda}, \frac{1}{\mu} M\right)=\frac{1}{\mu} V_{n-1,1}\left(K_{\lambda}, M\right)=1,
$$

so that

$$
\frac{1}{\mu} V_{n-1,1}(L, M)=V_{n-1,1}\left(L, \frac{1}{\mu} M\right) \leq 1=\frac{1}{\mu} V_{n-1,1}\left(K_{\lambda}, M\right) .
$$

In other words, $V_{n-1,1}(L, M) \leq V_{n-1,1}\left(K_{\lambda}, M\right)$ for all $M$.

But the argument above can be repeated, reversing the roles of $K_{\lambda}$ and $L$. To see this, recall the identity (11). It follows from (111) that for any value of $\lambda$, we have equality in the Brunn-Minkowski inequality for convex combinations of $K_{\lambda}$ and $L$. Fixing a value of $\lambda$, and setting $S_{\tau}=(1-\tau) L+\tau K_{\lambda}$, apply the same arguments as above using $L$ and $K_{\lambda}$ as the new "endpoints" to conclude that $V_{n-1,1}\left(K_{\lambda}, M\right) \leq V_{n-1,1}\left(S_{\tau}, M\right)$ for all $M$ and all $\tau \in[0,1]$. When $\tau=0$ this implies that $V_{n-1,1}\left(K_{\lambda}, M\right) \leq V_{n-1,1}(L, M)$ for all $M$. It now follows that

$$
V_{n-1,1}\left(K_{\lambda}, M\right)=V_{n-1,1}(L, M)
$$

for all $M$ and all $\lambda \in[0,1]$.

If $n=2$, set $\lambda=0$. Proposition 2.1 then implies that $K$ and $L$ are translates. This case is the starting point for induction on the dimension $n$.

If $n \geq 3$, then assume the theorem holds in lower dimensions. If $u$ is a unit vector, let $M$ be the line segment $\overline{o u}$ so that

$$
V_{n-1,1}\left(K_{\lambda}, \overline{o u}\right)=V_{n-1,1}(L, \overline{o u}) .
$$

It follows from (4) that

$$
V_{n-1}\left(\left(K_{\lambda}\right)_{u}\right)=V_{n-1}\left(L_{u}\right)
$$

for all $\lambda \in[0,1]$ so that

$$
V_{n-1}\left(K_{u}\right)=V_{n-1}\left((1-\lambda) K_{u}+\lambda L_{u}\right)=V_{n-1}\left(L_{u}\right) .
$$

Therefore,

$$
V_{n-1}\left((1-\lambda) K_{u}+\lambda L_{u}\right)^{\frac{1}{n-1}}=(1-\lambda) V_{n-1}\left(K_{u}\right)^{\frac{1}{n-1}}+\lambda V_{n-1}\left(L_{u}\right)^{\frac{1}{n-1}} .
$$

This is the equality case of the Brunn-Minkowski inequality in dimension $n-1$. Since $K$ and $L$ have interior, so do their projections (relative to $(n-1)$-dimensional subspaces). It now follows from the induction hypothesis that $K_{u}$ and $L_{u}$ are homothetic. Since $K$ and $L$ have homothetic projections in every direction $u$, it follows from Theorem 3.1 that $K$ and $L$ are homothetic. 


\section{REFERENCES}

[1] T. Bonnesen and W. Fenchel, Theory of Convex Bodies, BCS Associates, Moscow, Idaho, 1987. MR.920366 (88j:52001)

[2] C. Borell, Capacitary inequalities of the Brunn-Minkowski type, Math. Ann. 263 (1983), 179-184. MR698001 (84e:31005)

[3] H. Brascamp and E. Lieb, On extensions of the Brunn-Minkowski and Prékopa-Leindler theorems, including inequalities for log concave functions, and with an application to the diffusion equation, J. Functional Analysis 22 (1976), 366-389. MR0450480 (56:8774)

[4] G. Ewald, Combinatorial Convexity and Algebraic Geometry, Springer-Verlag, New York, 1996. MR 1418400 (97i:52012)

[5] R. J. Gardner, The Brunn-Minkowski inequality, Bull. Amer. Math. Soc. 39 (2002), 355-405. MR1898210 (2003f:26035)

[6] R. J. Gardner, Geometric Tomography (2nd Ed.), Cambridge University Press, Cambridge, 2006. MR2251886 (2007i:52010)

[7] R. J. Gardner and P. Gronchi, A Brunn-Minkowski inequality for the integer lattice, Trans. Amer. Math. Soc. 353 (2001), 3995-4024. MR1837217 (2002g:52011)

[8] H. Hadwiger, Seitenrisse konvexer Körper und Homothetie, Elem. Math. 18 (1963), 97-98. MR0155232 (27:5168)

[9] H. Hadwiger and D. Ohmann, Brunn-Minkowskischer Satz und Isoperimetrie, Math. Z. 66 (1956), 1-8. MR0082697(18:595c)

[10] R. Henstock and A. M. Macbeath, On the measure of sum sets, I. The theorems of Brunn, Minkowski and Lusternik, Proc. London Math. Soc. 3 (1953), 182-194. MR0056669 (15:109g)

[11] E. Lutwak, Intersection bodies and dual mixed volumes, Adv. Math. 71 (1988), 232-261. MR.963487 (90a:52023)

[12] E. Lutwak, The Brunn-Minkowski-Firey theory. I. Mixed volumes and the Minkowski problem, J. Differential Geom. 38 (1993), 131-150. MR1231704 (94g:52008)

[13] C. A. Rogers, Sections and projections of convex bodies, Portugal. Math. 24 (1965), 99-103. MR0198344 (33:6502)

[14] R. Schneider, Convex Bodies: The Brunn-Minkowski Theory, Cambridge University Press, Cambridge, 1993. MR1216521 (94d:52007)

[15] A. C. Thompson, Minkowski Geometry, Cambridge University Press, Cambridge, 1996. MR1406315 (97f:52001)

[16] R. Webster, Convexity, Oxford University Press, Cambridge, 1994. MR.1443208 (98h:52001)

Department of Mathematical Sciences, University of Massachusetts Lowell, Lowell, Massachusetts 01854

E-mail address: Daniel_Klain@uml.edu 